\title{
ADIABATIC SHEARING OF INCOMPRESSIBLE FLUIDS WITH TEMPERATURE-DEPENDENT VISCOSITY*
}

BY

\author{
C. M. DAFERMOS** AND L. HSIAO $\dagger$ \\ Brown-University
}

1. Introduction. In the framework of a program of investigating the stabilizing effects of various dissipative mechanisms in continuum thermomechanics, we have studied $[1,2]$ the initial-boundary value problem for the system of conservation laws of momentum and energy in one-dimensional thermoviscoelasticity. The techniques employed in the above papers restricted the analysis to the situation where viscosity does not vary with temperature; this assumption is rather crude, especially for fluid materials.

A convenient test problem for elucidating the effects of the dependence of viscosity on temperature is provided by adiabatic rectilinear shearing flow of an incompressible viscous fluid between two parallel plates. Indeed, in this case the conservation equations are particularly simple and viscosity is the sole dissipative mechanism present.

In a Cartesian coordinate system the two parallel plates occupy the planes $x=0$ and $x=1$. The flow is described by the velocity field $v(x, t)$ in the direction of the flow, perpendicular to the $x$-axis, and the temperature field $\theta(x, t)$. If we normalize units so that the density of the fluid is unity, the conservation equations of momentum and energy read

$$
v_{t}-\sigma_{x}=0, \quad e_{t}-\sigma v_{x}=0,
$$

where $\sigma$ is shear stress and $e$ is internal energy.

The fluid is assumed linearly viscous, that is

$$
\sigma=\mu v_{x},
$$

where $\mu$ is viscosity. Internal energy and viscosity are determined by temperature via known constitutive relations

$$
e=\hat{e}(\theta), \quad \mu=\hat{\mu}(\theta) .
$$

In typical fluids $\hat{e}(\theta)$ is increasing, convex and practically linear (i.e., constant specific heat) at moderate temperatures. The function $\hat{\mu}(\theta)$, on the other hand, is typically increasing in gases and decreasing in liquids. Several empirical or theoretical (derived within the context of the kinetic theory) forms of $\hat{\mu}(\theta)$ are recorded in the literature. For instance, the kinetic theory of gases with molecules behaving as ideal elastic spheres yields

$$
\mu=\alpha \theta^{1 / 2}
$$

* Received March 18, 1982. The authors are indebted to Professor Rodney Clifton for several illuminating discussions.

** The work of this author was supported by NSF grants MCS-79-05774-02, CME 80-23824 and by U. S. Army Contract ARO-DAAG-29-79-C-0161.

$\dagger$ On leave from the Mathematics Institute, Academia Sinica of China, Peking. 
while the standard form for liquids is

$$
\mu=\alpha \exp (\beta / \theta) .
$$

For our present purposes it is convenient to eliminate $\theta$ between $\hat{e}(\theta)$ and $\hat{\mu}(\theta)$ in (1.4) and visualize viscosity as a known smooth function of internal energy, $\mu=\mu(e)$, in which case the conservation equations (1.1), (1.2) take the form

$$
v_{t}-\left[\mu(e) v_{x}\right]_{x}=0, \quad e_{t}-\mu(e) v_{x}^{2}=0 .
$$

We assume that the fluid is subjected to steady shearing so that the boundary conditions read

$$
v(0, t)=0, \quad v(1, t)=1, \quad 0 \leq t<\infty .
$$

We also impose initial conditions

$$
v(x, 0)=v_{0}(x), \quad e(x, 0)=e_{0}(x), \quad 0 \leq x \leq 1,
$$

which are compatible with (1.9) in that $v_{0}(0)=0, v_{0}(1)=1$.

When $v_{0}(x)=x, e_{0}(x)=E_{0}=$ constant, the solution to (1.7), (1.8), (1.9), (1.10) describes a uniform shearing flow and can be written down explicitly:

$$
v(x, t)=x, \quad e(x, t)=E(t)
$$

where $E(t)$ is determined by

$$
\int_{E_{0}}^{E(t)} \frac{d e}{\mu(e)}=t .
$$

Our objective is to discuss the asymptotic stability of the solution (1.11).

As the fluid is being sheared, energy is pumped into it and, since the flow is adiabatic, the temperature will keep rising, tending to infinity with time. When, as $e \rightarrow \infty, \mu(e)$ approaches monotonically a positive finite constant $\mu(\infty)$, one expects that (1.7) will force $v_{x}$ to become asymptotically constant so that $(1.11)$ is asymptotically stable. To test this conjecture we first assume that $\mu(e)$ satisfies one of the following hypotheses:

$$
\begin{gathered}
\mu(e)>0, \quad \mu^{\prime}(e)>0, \quad\left[\mu^{2}(e)\right]^{\prime \prime} \leq 0, \quad \mu(\infty)<\infty, \quad 0<e<\infty, \\
\mu(e)>0, \quad \mu^{\prime}(e)<0, \quad \mu^{\prime \prime}(e) \geq 0, \quad \mu(\infty)>0, \quad 0<e<\infty,
\end{gathered}
$$

and show

THEOREM 1. Assume $v_{0}(x) \in W^{2,2}(0,1), e_{0}(x) \in W^{1,2}(0,1), e_{0}(x)>0,0 \leq x \leq 1$. Then, under hypothesis $(\mathrm{H} 1)$ or $(\mathrm{H} 2)$, there exists a unique classical solution of (1.7), (1.8), (1.9), $(1.10)$ on $[0,1] \times[0, \infty)$. Furthermore, as $t \rightarrow \infty$,

$$
\begin{aligned}
& v_{x}(x, t)=1+O\left(t^{-1}\right), \\
& \int_{e_{0}(x)}^{e(x, t)} \frac{d \xi}{\mu(\xi)}=t+O(1),
\end{aligned}
$$

uniformly in $x$ on $[0,1]$.

The situation is more interesting when $\mu(e)$ is allowed to tend to zero or infinity as $e \rightarrow \infty$. Indeed, in these cases it is conceivable that the asymptotic distribution of $v_{x}$, as dictated by (1.7), is not uniform. Still it is plausible that (1.11) is asymptotically stable provided $\mu(e)$ tends to zero or infinity in an "orderly" fashion. We verify this conjecture 
under the assumption that $\mu(e)$ satisfies one of the following hypotheses:

$$
\begin{gathered}
\mu(e)>0, \quad \mu^{\prime}(e) \geq 0, \quad 1 \leq-\frac{\mu(e) \mu^{\prime \prime}(e)}{\mu^{\prime}(e)^{2}} \leq v<\infty, \quad 0<e<\infty, \\
\mu(e)>0, \quad \mu^{\prime}(e) \leq 0, \quad 2<v \leq \frac{\mu(e) \mu^{\prime \prime}(e)}{\mu^{\prime}(e)^{2}} \leq N<\infty, \quad 0<e<\infty .
\end{gathered}
$$

A typical function class that satisfies $(\mathrm{H} 3)$ or $(\mathrm{H} 4)$ is

$$
\mu(e)=e^{\gamma}, \quad 0<\gamma \leq \frac{1}{2} \text { or }-1<\gamma<0 .
$$

THeorem 2. Assume $v_{0}(x) \in W^{2,2}(0,1), e_{0}(x) \in W^{1,2}(0,1), e_{0}(x)>0,0 \leq x \leq 1$. Then, under hypothesis $(\mathrm{H} 3)$ or $(\mathrm{H} 4)$, there exists a unique classical solution of (1.7), (1.8), (1.9), $(1.10)$ on $[0,1] \times[0, \infty)$. Furthermore, as $t \rightarrow \infty$,

$$
\begin{gathered}
v_{x}(x, t)=1+O\left(t^{-\eta}\right), \\
v_{t}(x, t)=O\left(t^{-1}\right), \\
\int_{e_{0}(x)}^{e(x, t)} \frac{d \xi}{\mu(\xi)}=t+O\left(t^{\delta}\right),
\end{gathered}
$$

uniformly in $x$ on $[0,1]$. In (1.16), $\eta=1+1 / v$ under (H3) and $\eta=1-2 / v$ under (H4). In (1.18), $\delta=0$ under $(\mathrm{H} 3)$ and $\delta=2 / v$ under $(\mathrm{H} 4)$.

We note that the precise growth assumptions on $\mu \mu^{\prime \prime} / \mu^{\prime 2}$ in (H3), (H4) are essential only for obtaining the exact decay rates in (1.16), (1.17), (1.18). These restrictions may be relaxed in various directions at the expense of settling for cruder decay rates for $v_{x}-1$ and $v_{t}$.

The proofs of Theorems 1 and 2 are presented in Sec. 3 and are based on a priori estimations. The estimates are obtained with the help of a number of identities for solutions of (1.7), (1.8), (1.9), (1.10), derived in Sec. 2, and certain properties of $\mu(e)$ which follow from (H3) or (H4) and are recorded in the Appendix.

2. Useful identities. Throughout this section, we assume that $(v(x, t), e(x, t))$ is a fixed classical solution of (1.7), (1.8), (1.9), (1.10) on $[0,1] \times[0, \infty)$ such that $v(\cdot, t), v_{x}(\cdot, t)$, $v_{t}(\cdot, t), v_{x x}(\cdot, t), e(\cdot, t), e_{x}(\cdot, t)$ are all in $C^{0}\left([0, \infty) ; L^{2}(0,1)\right)$ while $v_{x t}(\cdot, t)$ is in $C^{0}((0, \infty)$; $\left.L^{2}(0,1)\right)$ and $v_{t t}(\cdot, t)$ is in $L_{\text {loc }}^{2}\left((0, \infty) ; L^{2}(0,1)\right)$. We derive certain identities that will be used to estimate the solution in Sec. 3 .

On account of (1.3), (1.2) yields

$$
M(e)_{t}=\mu(e) e_{t}=\sigma^{2}
$$

where

$$
M(e) \stackrel{\operatorname{det}}{=} \int_{0}^{e} \mu(\xi) d \xi
$$

Using (1.1),

$$
\begin{aligned}
\sigma^{2}(x, t) & =\sigma^{2}(y, t)+2 \int_{y}^{x} \sigma(\xi, t) \sigma_{x}(\xi, t) d \xi \\
& =\int_{0}^{1} \sigma^{2}(y, t) d y+2 \int_{0}^{1} \int_{y}^{x} \sigma(\xi, t) v_{t}(\xi, t) d \xi d y
\end{aligned}
$$


so that $(2.1)$ gives

$$
\begin{aligned}
M(e(x, t))= & M\left(e_{0}(x)\right)+\int_{0}^{t} \int_{0}^{1} \sigma^{2}(y, \tau) d y d \tau \\
& +2 \int_{0}^{t} \int_{0}^{1} \int_{y}^{x} \sigma(\xi, \tau) v_{t}(\xi, \tau) d \xi d y d \tau .
\end{aligned}
$$

We now multiply $(1.7)$ by $v(x, t)$, integrate with respect to $x$ over $(0,1)$, integrate by parts and use the boundary conditions (1.9) to get

$$
\int_{0}^{1} v(x, t) v_{t}(x, t) d x+\int_{0}^{1} \mu(e(x, t)) v_{x}^{2}(x, t) d x=\sigma(1, t) .
$$

Integrating (2.5) over $(0, t)$ and using (1.8), we obtain

$$
\frac{1}{2} \int_{0}^{1} v^{2}(x, t) d x+\int_{0}^{1} e(x, t) d x=\int_{0}^{t} \sigma(1, \tau) d \tau+\frac{1}{2} \int_{0}^{1} v_{0}^{2}(x) d x+\int_{0}^{1} e_{0}(x) d x
$$

Next we multiply $(1.7)$ by $v_{t}$, integrate over $(0,1) \times(0, t)$, integrate by parts with respect to $x$ and $t$ and use (1.8) to deduce

$$
\begin{aligned}
\int_{0}^{t} \int_{0}^{1} v_{t}^{2} d x d \tau+\frac{1}{2} \int_{0}^{1} \mu(e(x, t)) v_{x}^{2}(x, t) d x-\frac{1}{2} \int_{0}^{t} \int_{0}^{1} \mu(e) \mu^{\prime}(e) v_{x}^{4} d x d \tau & =\frac{1}{2} \int_{0}^{1} \mu\left(e_{0}(x)\right) v_{0 x}^{2}(x) d x .
\end{aligned}
$$

Similarly, multiplying (1.7) by $t v_{t}$, following the same steps and using (1.8), we obtain

$$
\begin{aligned}
\int_{0}^{t} \int_{0}^{1} \tau v_{t}^{2} d x d \tau+\frac{1}{2} t \int_{0}^{1} \mu(e(x, t)) v_{x}^{2}(x, t) d x-\frac{1}{2} \int_{0}^{t} \int_{0}^{1} \tau \mu(e) \mu^{\prime}(e) v_{x}^{4} d x d \tau \\
-\frac{1}{2} \int_{0}^{1} e(x, t) d x=-\frac{1}{2} \int_{0}^{1} e_{0}(x) d x .
\end{aligned}
$$

To derive the next cycle of identities, we differentiate (1.7) with respect to $t$,

$$
v_{t t}-\left[\mu(e) v_{x t}+\mu(e) \mu^{\prime}(e) v_{x}^{3}\right]_{x}=0,
$$

and then multiply by $v_{t}$, integrate with respect to $x$ over $(0,1)$ and integrate by parts, thus arriving at

$$
\begin{aligned}
\frac{1}{2} \frac{d}{d t} \int_{0}^{1} v_{t}^{2}(x, t) d x+\int_{0}^{1} \mu(e(x, t)) v_{x t}^{2}(x, t) & d x \\
& +\int_{0}^{1} \mu(e(x, t)) \mu^{\prime}(e(x, t)) v_{x}^{3}(x, t) v_{x t}(x, t) d x=0 .
\end{aligned}
$$

Integrating (2.10) over $(0, t)$ and integrating by parts, we arrive at

$$
\begin{aligned}
& \frac{1}{2} \int_{0}^{1} v_{t}^{2}(x, t) d x+\int_{0}^{t} \int_{0}^{1} \mu(e) v_{x t}^{2} d x d \tau+\frac{1}{4} \int_{0}^{1} \mu(e(x, t)) \mu^{\prime}(e(x, t)) v_{x}^{4}(x, t) d x \\
&-\frac{1}{8} \int_{0}^{t} \int_{0}^{1}\left[\mu^{2}(e)\right]^{\prime \prime} \mu(e) v_{x}^{6} d x d \tau=\frac{1}{2} \int_{0}^{1} v_{t}^{2}(x, 0) d x \\
&+\frac{1}{4} \int_{0}^{1} \mu\left(e_{0}(x)\right) \mu^{\prime}\left(e_{0}(x)\right) v_{0 x}^{4}(x) d x
\end{aligned}
$$


We now multiply (2.10) by $t^{2}$ and then integrate over $(0, t)$ to obtain

$$
\begin{array}{r}
\frac{1}{2} t^{2} \int_{0}^{1} v_{t}^{2}(x, t) d x-\int_{0}^{t} \int_{0}^{1} \tau v_{t}^{2} d x d \tau+\int_{0}^{t} \int_{0}^{1} \tau^{2} \mu(e) v_{x t}^{2} d x d \tau \\
+\int_{0}^{t} \int_{0}^{1} \tau^{2} \mu(e) \mu^{\prime}(e) v_{x}^{3} v_{x t} d x d \tau=0 .
\end{array}
$$

Finally, we multiply $(2.9)$ by $t^{3} v_{t t}$, integrate over $(0,1) \times(0, t)$ and perform a number of integrations by parts with respect to $x$ and $t$, thus obtaining

$$
\begin{aligned}
& \int_{0}^{t} \int_{0}^{1} \tau^{3} v_{t t}^{2} d x d \tau+\frac{1}{2} t^{3} \int_{0}^{1} \mu(e(x, t)) v_{x t}^{2}(x, t) d x=\frac{3}{2} \int_{0}^{t} \int_{0}^{1} \tau^{2} \mu(e) v_{x t}^{2} d x d \tau \\
& +\frac{7}{2} \int_{0}^{t} \int_{0}^{1} \tau^{3} \mu(e) \mu^{\prime}(e) v_{x}^{2} v_{x t}^{2} d x d \tau-t^{3} \int_{0}^{1} \mu(e(x, t)) \mu^{\prime}(e(x, t)) v_{x}^{3}(x, t) v_{x t}(x, t) d x \\
& +3 \int_{0}^{t} \int_{0}^{1} \tau^{2} \mu(e) \mu^{\prime}(e) v_{x}^{3} v_{x t} d x d \tau+\frac{1}{2} \int_{0}^{t} \int_{0}^{1} \tau^{3}\left[\mu^{2}(e)\right]^{\prime \prime} \mu(e) v_{x}^{5} v_{x t} d x d \tau
\end{aligned}
$$

3. Proof of Theorems. In this section, we consider the solution $(v(x, t), e(x, t))$ discussed in Sec. 2 and use the identities derived there in order to establish the estimates that will yield the proofs of Theorems 1 and 2 stated in the introduction. In what follows, $K$ will stand for a generic constant which can be estimated from above solely in terms of properties of the function $\mu(e)$ and upper bounds of the $W^{2,2}(0,1)$ norm of $v_{0}(x)$ and the $W^{1,2}(0,1)$ norm of $e_{0}(x)$.

Lemma 3.1. Under hypothesis (H1), (H2), (H3), or (H4),

$$
\int_{0}^{t} \int_{0}^{1} v_{t}^{2} d x d \tau \leq K, \quad 0 \leq t<\infty .
$$

Proof. Under hypothesis (H2) or (H4), (3.1) follows directly from (2.7). For the hypothesis $(\mathrm{H} 1)$ or $(\mathrm{H} 3),(3.1)$ is a consequence of $(2.11)$ upon noting that $\left[\mu^{2}(e)\right]^{\prime \prime} \leq 0$ and

$$
\int_{0}^{t} \int_{0}^{1} v_{t}^{2} d x d \tau \leq \max _{0 \leqslant x \leqslant 1} \frac{1}{\mu\left(e_{0}(x)\right)} \int_{0}^{t} \int_{0}^{1} \mu(e) v_{x t}^{2} d x d \tau .
$$

We now define

$$
\varphi(t)=1+\int_{0}^{t} \int_{0}^{1} \sigma^{2} d x d \tau, \quad 0 \leq t<\infty .
$$

Lemma 3.2. Under hypothesis (H1), (H2), (H3), or (H4),

$$
\frac{1}{K} \varphi(t) \leq M(e(x, t)) \leq K \varphi(t), \quad 0 \leq x \leq 1, \quad 0 \leq t<\infty .
$$

Proof. Because of (2.4), (3.1) and (3.3),

$$
\begin{aligned}
& M(e(x, t)) \leq 2 \int_{0}^{t} \int_{0}^{1} \sigma^{2}(y, \tau) d y d \tau+K_{1} \leq\left(K_{1}+2\right) \varphi(t), \\
& M(e(x, t)) \geq \frac{1}{2} \int_{0}^{t} \int_{0}^{1} \sigma^{2}(y, \tau) d y d \tau-K_{2}=\frac{1}{2} \varphi(t)-K_{3} .
\end{aligned}
$$


At the same time,

$$
M(e(x, t)) \geq \min _{0 \leqslant x \leqslant 1} M\left(e_{0}(x)\right) \stackrel{\text { def }}{=} 1 / K_{4} .
$$

Combining (3.6) with (3.7), we easily deduce

$$
M(e(x, t)) \geq \frac{1}{2\left(1+K_{3} K_{4}\right)} \varphi(t)
$$

so that (3.4) follows from (3.5) and (3.8).

Let us now set

$$
E(t)=M^{-1}\left(\frac{1}{K} \varphi(t)\right) .
$$

From (3.4) and properties of the functions $\mu(e), \mu^{\prime}(e)$, and $M^{-1}(e)$ (see Appendix, Eqs. (4.2), (4.3), (4.4) and (4.5)), we deduce

Corollary 3.1. Under hypothesis (H1), (H2), (H3), or (H4),

$$
\begin{gathered}
E(t) \leq e(x, t) \leq K E(t), \quad 0 \leq x \leq 1, \quad 0 \leq t<\infty, \\
\frac{1}{K} \mu(E(t)) \leq \mu(e(x, t)) \leq K \mu(E(t)), \quad 0 \leq x \leq 1, \quad 0 \leq t<\infty, \\
\left|\mu^{\prime}(e(x, t))\right| \leq\left|\mu^{\prime}(E(t))\right|, \quad 0 \leq x \leq 1, \quad 0 \leq t<\infty .
\end{gathered}
$$

We now proceed to the estimation of the mean square growth of stress.

LEMma 3.3. Under hypothesis (H1), (H2), (H3), or (H4),

$$
\frac{1}{K} \mu^{2}(E(t)) \leq \int_{0}^{1} \sigma^{2}(x, t) d x \leq K \mu^{2}(E(t)), \quad 0 \leq t<\infty .
$$

Proof. Using (1.3), (3.11), and (1.9), we obtain

$$
\begin{aligned}
\int_{0}^{1} \sigma^{2}(x, t) d x & \geq \frac{1}{K_{1}} \mu^{2}(E(t)) \int_{0}^{1} v_{x}^{2}(x, t) d x \geq \frac{1}{K_{1}} \mu^{2}(E(t))\left[\int_{0}^{1} v_{x}(x, t) d x\right]^{2} \\
& =\frac{1}{K_{1}} \mu^{2}(E(t))
\end{aligned}
$$

which establishes the left half of (3.13). For the right half of (3.13) we have to distinguish two cases.

Assume first that $(\mathrm{H} 1)$ or $(\mathrm{H} 3)$ holds. By the maximum principle for the parabolic equation (1.7), $|v(x, t)| \leq \max _{0 \leq y \leq 1}\left|v_{0}(y)\right|, 0 \leq x \leq 1,0 \leq t<\infty$. At the same time, by (2.11),

$$
\int_{0}^{1} v_{t}^{2}(x, t) d x \leq K_{2}, \quad 0 \leq t<\infty .
$$

Hence, combining (1.3), (3.11), (2.5), (3.15), (1.1), and (3.14),

$$
\begin{aligned}
\left\{\int_{0}^{1} \sigma^{2}(x, t) d x\right\}^{2} & \leq K_{3} \mu^{2}(E(t))\left\{\int_{0}^{1} \mu(e(x, t)) v_{x}^{2}(x, t) d x\right\}^{2} \\
& \leq K_{3} \mu^{2}(E(t))\left\{2 \sigma^{2}(1, t)+K_{4}\right\}
\end{aligned}
$$




$$
\begin{aligned}
& \leq K_{3} \mu^{2}\left(E(t)\left\{4 \int_{0}^{1} \sigma^{2}(x, t) d x+2 \int_{0}^{1} \sigma_{x}^{2}(x, t) d x+K_{4}\right\}\right. \\
& \leq K_{3} \mu^{2}(E(t))\left\{4 \int_{0}^{1} \sigma^{2}(x, t) d x+\frac{K_{5}}{\mu^{2}(E(t))} \int_{0}^{1} \sigma^{2}(x, t) d x\right\} \\
& \leq K_{6} \mu^{2}(E(t)) \int_{0}^{1} \sigma^{2}(x, t) d x,
\end{aligned}
$$

whence the right half of (3.13) follows.

We now take up the case of (H2) or (H4) for $t \geq 1$. Using (3.10), (2.6), (3.3), (3.1), (3.9) and Schwarz's inequality,

$$
\begin{aligned}
E^{2}(t) & \leq\left\{\int_{0}^{1} e(x, t) d x\right\}^{2} \leq 2\left\{\int_{0}^{t} \sigma(1, \tau) d \tau\right\}^{2}+K_{7} \\
& \leq 2 t \int_{0}^{t} \sigma^{2}(1, \tau) d \tau+K_{7} \\
& \leq 2 t \int_{0}^{t}\left\{2 \int_{0}^{1} \sigma^{2}(x, \tau) d x+\int_{0}^{1} \sigma_{x}^{2}(x, \tau) d x\right\} d \tau+K_{7} \\
& \leq K_{8} t \varphi(t) \leq K_{9} t M(E(t)) .
\end{aligned}
$$

On the other hand, by (1.3), (3.11), (2.8) and (3.10),

$$
\begin{aligned}
\int_{0}^{1} \sigma^{2}(x, t) d x & \leq K_{10} \mu(E(t)) \int_{0}^{1} \mu(e(x, t)) v_{x}^{2}(x, t) d x \\
& \leq \frac{K_{11}}{t} \mu(E(t))\left\{\int_{0}^{1} e(x, t) d x+K_{12}\right\} \leq \frac{K_{13}}{t} \mu(E(t)) E(t) .
\end{aligned}
$$

Combining (3.17) with (3.18), we obtain

$$
\int_{0}^{1} \sigma^{2}(x, t) d x \leq K_{14} \frac{M(E(t))}{E(t) \mu(E(t))} \mu^{2}(E(t)) .
$$

Applying L'Hopital's rule twice and using (H4), we find

$$
\begin{aligned}
\liminf _{e \rightarrow \infty} \frac{e \mu(e)}{M(e)} & =1+\liminf _{e \rightarrow \infty} \frac{e}{\mu(e) / \mu^{\prime}(e)} \\
& =1+\liminf _{e \rightarrow \infty} \frac{\mu^{\prime 2}(e)}{\mu^{\prime 2}(e)-\mu(e) \mu^{\prime \prime}(e)} \geq \frac{v-2}{v-1}
\end{aligned}
$$

Therefore,

$$
\frac{e \mu(e)}{M(e)} \geq \frac{1}{K_{15}}>0, \quad 0<e<\infty .
$$

In view of (3.21), (3.19) yields the right half of (3.13), for $t \geq 1$, under hypothesis (H2) or (H4). 
It remains to establish the right half of (3.13) on $0 \leq t<1$, for the case (H2) or (H4). To this end we first note that, by virtue of $(2.7)$,

$$
\int_{0}^{1} \sigma^{2}(x, t) d x \leq \max _{0 \leqslant y \leqslant 1} \mu\left(e_{0}(y)\right) \int_{0}^{1} \mu(e(x, t)) v_{x}^{2}(x, t) d x \leq K_{16} .
$$

Thus (3.3), (3.9) yield $\varphi(t) \leq 1+K_{16}, E(t) \leq K_{17}, 0 \leq t \leq 1$, and thereby

$$
\mu(E(t)) \geq \frac{1}{K_{18}}, \quad 0 \leq t<1 .
$$

It is now clear that (3.22) together with (3.23) imply the right half of (3.13) on $0 \leq t<1$, and this completes the proof of the lemma.

Lemma 3.3 allows us to estimate the growth of $E(t)$ in time. To see this note that, on account of (3.9), (2.2) and (3.3),

$$
\frac{d E(t)}{d t}=\frac{1}{K} \frac{1}{\mu(E(t))} \frac{d \varphi(t)}{d t}=\frac{1}{K} \frac{1}{\mu(E(t))} \int_{0}^{1} \sigma^{2}(x, t) d x .
$$

Hence, using (3.13), we arrive at the following

Corollary 3.2. Under hypothesis (H1), (H2), (H3), or (H4),

$$
\begin{gathered}
\frac{1}{K} \mu(E(t)) \leq \frac{d E(t)}{d t} \leq K \mu(E(t)), \quad 0<t<\infty, \\
\frac{1}{K} t \leq \int_{E(0)}^{E(t)} \frac{d e}{\mu(e)} \leq K t, \quad 0 \leq t<\infty .
\end{gathered}
$$

Lemma 3.4. Under hypothesis $(\mathrm{H} 1)$ or $(\mathrm{H} 2)$,

$$
\int_{0}^{1} v_{t}^{2}(x, t) d x \leq K\left\{\exp \left[-\frac{1}{K_{0}} E(t)\right]+\mu^{\prime 2}\left(\frac{1}{2} E(t)\right)\right\}, \quad 0 \leq t<\infty,
$$

while under hypothesis $(\mathrm{H} 3)$ or $(\mathrm{H} 4)$,

$$
\int_{0}^{1} v_{t}^{2}(x, t) d x \leq K \mu^{\prime 2}(E(t)), \quad 0 \leq t<\infty .
$$

Proof. Upon using Schwarz's inequality, (2.10) yields

$$
\frac{d}{d t} \int_{0}^{1} v_{t}^{2}(x, t) d x+\int_{0}^{1} \mu(e(x, t)) v_{x t}^{2}(x, t) d x \leq \int_{0}^{1} \mu(e(x, t)) \mu^{\prime 2}(e(x, t)) v_{x}^{6}(x, t) d x .
$$

Therefore, by virtue of (3.11), (3.12), (1.3), (3.13) and

$$
\int_{0}^{1} \sigma_{x}^{2}(x, t) d x=-\int_{0}^{1} \sigma(x, t) \sigma_{x x}(x, t) d x
$$

we deduce from (3.28)

$$
\begin{gathered}
\frac{d}{d t} \int_{0}^{1} v_{t}^{2}(x, t) d x+\frac{2}{K_{1}} \mu(E(t)) \int_{0}^{1} v_{x t}^{2}(x, t) d x \leq K_{2} \frac{\mu^{\prime 2}(E(t))}{\mu^{5}(E(t))} \int_{0}^{1} \sigma^{6}(x, t) d x \\
\leq K_{2} \frac{\mu^{\prime 2}(E(t))}{\mu^{5}(E(t))}\left[\max _{0 \leqslant x \leqslant 1} \sigma^{2}(x, t)\right]^{2} \int_{0}^{1} \sigma^{2}(x, t) d x
\end{gathered}
$$




$$
\begin{aligned}
& \leq K_{3} \frac{\mu^{\prime 2}(E(t))}{\mu^{3}(E(t))}\left\{\int_{0}^{1} \sigma^{2}(x, t) d x+2\left[\int_{0}^{1} \sigma^{2}(x, t) d x\right]^{1 / 2}\left[\int_{0}^{1} \sigma_{x}^{2}(x, t) d x\right]^{1 / 2}\right\}^{2} \\
& \leq K_{3} \frac{\mu^{\prime 2}(E(t))}{\mu^{3}(E(t))}\left\{\int_{0}^{1} \sigma^{2}(x, t) d x+2\left[\int_{0}^{1} \sigma^{2}(x, t) d x\right]^{3 / 4}\left[\int_{0}^{1} \sigma_{x x}^{2}(x, t) d x\right]^{1 / 4}\right\}^{2} \\
& \leq K_{4} \mu(E(t)) \mu^{\prime 2}(E(t))+K_{5} \mu^{\prime 2}(E(t))\left[\int_{0}^{1} v_{x t}^{2}(x, t) d x\right]^{1 / 2} \\
& \leq\left\{K_{4}+\frac{1}{4} K_{1} K_{5}^{2}\left[\frac{\mu^{\prime}(E(t))}{\mu(E(t))}\right]^{2}\right\} \mu(E(t)) \mu^{\prime 2}(E(t))+\frac{1}{K_{1}} \mu(E(t)) \int_{0}^{1} v_{x t}^{2}(x, t) d x .
\end{aligned}
$$

Keeping in mind that $\mu^{\prime}(e) / \mu(e)$ is bounded on $[E(0), \infty),(3.30)$ gives

$$
\frac{d}{d t} \int_{0}^{1} v_{t}^{2}(x, t) d x+\frac{1}{K_{1}} \mu(E(t)) \int_{0}^{1} v_{t}^{2}(x, t) d x \leq K_{6} \mu(E(t)) \mu^{\prime 2}(E(t)) .
$$

Integrating the differential inequality (3.31), we obtain

$$
\begin{aligned}
& \int_{0}^{1} v_{t}^{2}(x, t) d x \leq \exp \left\{-\frac{1}{K_{1}} \int_{0}^{t} \mu(E(\tau)) d \tau\right\} \int_{0}^{1} v_{t}^{2}(x, 0) d x \\
& \quad+K_{6} \int_{0}^{t} \mu(E(s)) \mu^{\prime 2}(E(s)) \exp \left\{-\frac{1}{K_{1}} \int_{s}^{t} \mu(E(\tau)) d \tau\right\} d s .
\end{aligned}
$$

Recalling (3.25), (3.32) yields

$$
\begin{aligned}
\int_{0}^{1} v_{t}^{2}(x, t) d x \leq & \exp \left\{\frac{1}{K_{7}}[E(0)-E(t)]\right\} \int_{0}^{1} v_{t}^{2}(x, 0) d x \\
& +K_{8} \int_{E(0)}^{E(t)} \mu^{\prime 2}(e) \exp \left\{\frac{1}{K_{7}}[e-E(t)]\right\} d e \\
\leq & K_{9}\left\{1+\int_{E(0)}^{E(t)} \mu^{\prime 2}(e) \exp \left[\frac{1}{K_{7}} e\right] \operatorname{de}\right\} \exp \left[-\frac{1}{K_{7}} E(t)\right] .
\end{aligned}
$$

Under $(\mathrm{H} 1)$ or $(\mathrm{H} 2)$,

$$
\int_{E(0)}^{E(t)} \mu^{\prime 2}(e) \exp \left[\frac{1}{K_{7}} e\right] d e \leq K_{10} \exp \left[\frac{1}{2 K_{7}} E(t)\right]+K_{11} \mu^{\prime 2}\left(\frac{1}{2} E(t)\right) \exp \left[\frac{1}{K_{7}} E(t)\right]
$$

so that (3.33) yields (3.27).

We now turn to the case where $(\mathrm{H} 3)$ or $(\mathrm{H} 4)$ holds. Noting that $\mu^{\prime \prime}(e) / \mu^{\prime}(e) \rightarrow 0$, as $e \rightarrow \infty$, and applying L'Hopital's rule:

$$
\lim _{e \rightarrow \infty} \frac{\int_{0}^{e} \mu^{\prime 2}(\xi) \exp \left[\frac{1}{K_{7}} \xi\right] d \xi}{\mu^{\prime 2}(e) \exp \left[\frac{1}{K_{7}} e\right]}=\lim _{e \rightarrow \infty} \frac{\mu^{\prime 2}(e) \exp \left[\frac{1}{K_{7}} e\right]}{\left[2 \mu^{\prime}(e) \mu^{\prime \prime}(e)+\frac{1}{K_{7}} \mu^{\prime 2}(e)\right] \exp \left[\frac{1}{K_{7}} e\right]}=K_{7} .
$$

Therefore, (3.28) follows from (3.33). 
LEMma 3.5. Under hypothesis (H1), (H2), or (H3),

$$
\int_{0}^{1}\left|e_{x}(x, t)\right| d x \leq K, \quad 0 \leq t<\infty,
$$

while under hypothesis (H4),

$$
\int_{0}^{1}\left|e_{x}(x, t)\right| d x \leq \frac{K}{\mu(E(t))}, \quad 0 \leq t<\infty .
$$

Proof. Differentiating (2.4) with respect to $x$ yields

$$
\mu(e(x, t)) e_{x}(x, t)=M\left(e_{0}(x)\right)_{x}+2 \int_{0}^{t} \sigma(x, \tau) v_{t}(x, \tau) d \tau .
$$

By virtue of (3.13) and (3.25),

$$
\begin{gathered}
\int_{0}^{1}\left|\int_{0}^{t} \sigma(x, \tau) v_{t}(x, \tau) d \tau\right| d x \leq \int_{0}^{t}\left\{\int_{0}^{1} \sigma^{2}(x, \tau) d x\right\}^{1 / 2}\left\{\int_{0}^{1} v_{t}^{2}(x, \tau) d x\right\}^{1 / 2} d \tau \\
\leq K_{1} \int_{0}^{t} \mu(E(\tau))\left\{\int_{0}^{1} v_{t}^{2}(x, \tau) d x\right\}^{1 / 2} d \tau \leq K_{2} \int_{E(0)}^{E(t)}\left\{\int_{0}^{1} v_{t}^{2}(x, \tau) d x\right\}^{1 / 2} d E(\tau) .
\end{gathered}
$$

We now estimate the integral on the right-hand side of (3.39) using (3.27), under hypothesis $(\mathrm{H} 1)$ or $(\mathrm{H} 2)$, and (3.28), under hypothesis $(\mathrm{H} 3)$ or $(\mathrm{H} 4)$. Combining the result with (3.38) and using (3.11), we arrive at (3.36) and (3.37).

LEMMA 3.6. Under hypothesis (H1) or (H2),

$$
\int_{0}^{1}\left|v_{x x}(x, t)\right| d x \leq K\left\{\exp \left[-\frac{1}{2 K_{0}} E(t)\right]+\left|\mu^{\prime}\left(\frac{1}{2} E(t)\right)\right|\right\}, \quad 0 \leq t<\infty ;
$$

under hypothesis $(\mathrm{H} 3)$,

$$
\int_{0}^{1}\left|v_{x x}(x, t)\right| d x \leq K \frac{\mu^{\prime}(E(t))}{\mu(E(t))}, \quad 0 \leq t<\infty ;
$$

under hypothesis (H4),

$$
\int_{0}^{1}\left|v_{x x}(x, t)\right| d x \leq-K \frac{\mu^{\prime}(E(t))}{\mu^{2}(E(t))}, \quad 0 \leq t<\infty .
$$

Proof. From (1.3),

$$
v_{x x}=\frac{\sigma_{x}}{\mu(e)}-\frac{\sigma \mu^{\prime}(e) e_{x}}{\mu^{2}(e)} .
$$

We note that, by account of (3.13), (3.27) and (3.28),

$$
|\sigma(x, t)| \leq K \mu(e(x, t)), \quad 0 \leq x \leq 1, \quad 0 \leq t<\infty .
$$

Combining (3.44), (3.11), (3.12), (3.27), (3.28), (3.36), and (3.37), we arrive at (3.40), (3.41) and (3.42). 
Since

$$
\left|v_{x}(y, t)-1\right| \leq \int_{0}^{1}\left|v_{x x}(x, t)\right| d x, \quad 0 \leq y \leq 1, \quad 0 \leq t<\infty,
$$

Lemma 3.6 yields decay rates for $v_{x}(x, t)-1$.

LEMMA 3.7. Under hypothesis (H1), (H2), or (H3),

$$
\left|\int_{e_{0}(x)}^{e(x, t)} \frac{d e}{\mu(e)}-t\right| \leq K, \quad 0 \leq x \leq 1, \quad 0 \leq t<\infty,
$$

while under hypothesis (H4),

$$
\left|\int_{e_{0}(x)}^{e(x, t)} \frac{d e}{\mu(e)}-t\right| \leq \frac{K}{\mu^{2}(E(t))}, \quad 0 \leq x \leq 1, \quad 0 \leq t<\infty .
$$

Proof. Using (1.2), (1.3), (3.45) and (3.25) yields

$$
\begin{aligned}
& \left|\int_{e^{0}(x)}^{e(x, t)} \frac{d e}{\mu(e)}-t\right|=\left|\int_{0}^{t}\left[v_{x}^{2}(x, \tau)-1\right] d \tau\right| \leq K_{1} \int_{0}^{t}\left|v_{x}(x, \tau)-1\right| d \tau \\
& \quad \leq K_{2} \int_{0}^{t} \int_{0}^{1}\left|v_{x x}(x, \tau)\right| d x d \tau \leq K_{3} \int_{E(0)}^{E(t)} \int_{0}^{1}\left|v_{x x}\right| d x \frac{d E(\tau)}{\mu(E(\tau))} .
\end{aligned}
$$

Therefore, combining (3.48) with (3.40), (3.41) or (3.42), we deduce (3.46) and (3.47).

Our next project is to express the decay rates obtained in the above propositions in terms of $t$. To this end we employ (3.26) and the assumed properties of the function $\mu(e)$ and its derivatives.

We first consider the case (H1) or (H2) holds. By (3.26), $(1 / K) t \leq E(t) \leq K t$, for large $t$. At the same time, in view of

$$
e \mu^{\prime}(e)=\int_{0}^{e} \xi \mu^{\prime \prime}(\xi) d \xi+\mu(e)-\mu(0), \quad 0<e<\infty,
$$

we deduce

$$
\left|\mu^{\prime}(e)\right| \leq K / e, \quad 0<e<\infty \text {. }
$$

Therefore, (1.13) follows from (3.45), (3.40) and (3.50). The remaining estimate has already been established by (3.46).

We now turn to the case of hypothesis (H3) or (H4). We first use L'Hopital's rule

$$
\lim _{e \rightarrow \infty} \sup _{e \rightarrow f} \frac{\frac{1}{\mu^{\prime}(e)}}{\int^{e} \frac{d \xi}{\mu(\xi)}}=-\lim _{e \rightarrow \infty} \sup \frac{\mu(e) \mu^{\prime \prime}(e)}{\mu^{\prime 2}(e)}
$$

in conjunction with (H3), (H4) and (3.26) to infer

$$
\frac{1}{K} t \leq \frac{1}{\left|\mu^{\prime}(E(t))\right|} \leq K t, \quad 1 \leq t<\infty .
$$

Let us fix our attention to (H3). We note that (3.46) yields (1.18) with $\delta=0$. To get (1.16), we observe that $(\mathrm{H} 3)$ implies $\left[\mu^{v+1}(e)\right]^{\prime \prime} \geq 0$; hence $\left[\mu^{v+1}(e)\right]^{\prime}$ is bounded from 
below, say $\mu^{v}(e) \mu^{\prime}(e) \geq 1 / K, e>0$. Then, by (3.52),

$$
\mu(E(t)) \geq \frac{1}{K} t^{1 / v}, \quad 1 \leq t<\infty,
$$

so that (3.45), (3.41) yield (1.16) with $\eta=1+1 / v$.

Next we consider the case (H4). (H4) implies $\left[\mu^{1-v}(e)\right]^{\prime \prime} \leq 0$ so $\left[\mu^{1-v}(e)\right]^{\prime}$ is bounded from above, say $(1-v) \mu^{-v}(e) \mu^{\prime}(e) \leq K, e>0$. It then follows from (3.52) that

$$
\mu(E(t)) \geq \frac{1}{K} t^{-1 / v}, \quad 1 \leq t<\infty .
$$

Combining (3.52), (3.54), (3.45), (3.42) and (3.47), we arrive at (1.16) and (1.18) with $\eta=1-2 / v$ and $\delta=2 / v$.

The remaining estimate (1.17) is an immediate corollary of (3.28), (3.52) and the following proposition:

Lemma 3.8. Under hypothesis $(\mathrm{H} 3)$ or $(\mathrm{H} 4)$,

$$
\int_{0}^{1} v_{x t}^{2}(x, t) d x \leq K / t^{2}, \quad 0<t<\infty .
$$

Proof. From (2.12) and Schwarz's inequality,

$$
\int_{0}^{t} \int_{0}^{1} \tau^{2} \mu(e) v_{x t}^{2} d x d \tau \leq 2 \int_{0}^{t} \int_{0}^{1} \tau v_{t}^{2} d x d \tau+\int_{0}^{t} \int_{0}^{1} \tau^{2} \mu(e) \mu^{\prime 2}(e) v_{x}^{6} d x d \tau
$$

To estimate the right-hand side of (3.56), we first note that, by virtue of (3.28), (3.52),

$$
\int_{0}^{1} v_{t}^{2}(x, t) d x \leq K_{1} \mu^{\prime 2}(E(t)) \leq K_{2} \mu(E(t)) \mu^{\prime}(E(t)) \leq \frac{K_{3}}{t} \mu(E(t))
$$

and then use (3.57), (3.11), (3.12), (3.52), and (1.16) to get

$$
\int_{0}^{t} \int_{0}^{1} \tau^{2} \mu(e) v_{x t}^{2} d x d \tau \leq K_{4} \int_{0}^{t} \mu(E(\tau)) d \tau .
$$

For the remainder of the proof we employ (2.13). We note that, in view of $(\mathrm{H} 3)$ or $(\mathrm{H} 4)$, $\left|\left[\mu^{2}(e)\right]^{\prime \prime}\right| \leq K_{5} \mu^{\prime 2}(e)$. Taking account of (3.11), (3.12), (3.52), (1.16), (3.58), (3.25) and applying Schwarz's inequality a number of times on (2.13), we deduce

$$
\begin{aligned}
t^{3} \mu(E(t)) \int_{0}^{1} v_{x t}^{2}(x, t) d x & \leq K_{6} t \mu(E(t))+K_{7} \int_{0}^{t} \mu(E(\tau)) d \tau \\
& \leq K_{6} t \mu(E(t))+K_{8} E(t),
\end{aligned}
$$

whence

$$
\int_{0}^{1} v_{x t}^{2}(x, t) d x \leq \frac{K_{6}}{t^{2}}+\frac{K_{8}}{t^{2}} \frac{E(t)}{t \mu(E(t))} .
$$

By virtue of (3.26), in order to show that $E(t) /[t \mu(E(t))]$ is bounded it suffices to prove that

$$
\frac{\frac{e}{\mu(e)}}{\int^{e} \frac{d \xi}{\mu(\xi)}}
$$


is bounded, $0<e<\infty$. Under hypothesis (H3), the function (3.61) is obviously bounded by 1 . For the case (H4), we apply L'Hopital's rule twice to get

$$
\begin{aligned}
\limsup _{e \rightarrow \infty} \frac{\frac{e}{\mu(e)}}{\int \frac{d \xi}{\mu(\xi)}} & =1+\limsup _{e \rightarrow \infty} \frac{e}{-\frac{\mu(e)}{\mu^{\prime}(e)}} \\
& =1+\limsup _{e \rightarrow \infty} \frac{\mu^{\prime 2}(e)}{\mu(e) \mu^{\prime \prime}(e)-\mu^{\prime 2}(e)} \leq \frac{v}{v-1} .
\end{aligned}
$$

Thus the function (3.61) is bounded and (3.60) implies (3.55).

We have thus established a priori the decay estimates in Theorems 1 and 2. The proof that a solution exists can now be obtained by a routine procedure. One first establishes the existence of a local solution on a maximal time interval by means of a straightforward contraction argument and then uses the derived a priori estimates to show that this solution cannot escape in finite time.

Appendix. We record here certain useful properties of the functions $\mu(e)$ and $M^{-1}(s)$ that are induced by the hypotheses $(\mathrm{H} 3)$ and $(\mathrm{H} 4)$.

We first note that if $f(\xi)$ is a smooth, nonnegative, increasing and concave function on $[0, \infty)$, i.e., $f(\xi) \geq 0, f^{\prime}(\xi)>0, f^{\prime \prime}(\xi) \leq 0$, then

$$
1 \leq \frac{f(k \xi)}{f(\xi)} \leq k, \quad 0<\xi<\infty, \quad k \geq 1 .
$$

These assumptions are satisfied, under hypothesis (H3), by the functions $\mu(e)$ and $M^{-1}(s)$, so we have

$$
\begin{aligned}
& 1 \leq \frac{\mu(k e)}{\mu(e)} \leq k, \quad 0<e<\infty, \quad 1 \leq k<\infty, \\
& 1 \leq \frac{M^{-1}(k s)}{M^{-1}(s)} \leq k, \quad 0<s<\infty, \quad 1 \leq k<\infty .
\end{aligned}
$$

Under hypothesis (H4) the above assumptions hold for the function $\mu^{1-v}(e)$, so we deduce

$$
1 \geq \frac{\mu(k e)}{\mu(e)} \geq k^{1 /(1-v)}, \quad 0<e<\infty, \quad 1 \leq k<\infty .
$$

Finally, we establish the analogue of (4.3) under hypothesis (H4), namely,

$$
1 \leq M^{-1}(k s) / M^{-1}(s) \leq \lambda(k), \quad 0<s<\infty, \quad 1 \leq k<\infty .
$$

To this end let us define

$$
g(e, \lambda)=M(\lambda e) / M(e), \quad 0<e<\infty, \quad 1 \leq \lambda<\infty .
$$

On account of (3.21) and (4.4),

$$
\frac{\partial g(e, \lambda)}{\partial \lambda}=\frac{e \mu(\lambda e)}{M(e)}=\frac{e \mu(e)}{M(e)} \frac{\mu(\lambda e)}{\mu(e)} \geq \frac{1}{K_{15}} \lambda^{1 /(1-v)}
$$

whence

$$
g(e, \lambda) \geq g(e, 1)+\frac{1}{K_{15}} \int_{1}^{\lambda} \xi^{1 /(1-v)} d \xi=1+\frac{1}{K_{15}} \frac{v-1}{v-2}\left(\lambda^{(v-2) /(v-1)}-1\right) .
$$


For $k \geq 1$ we define

$$
\lambda(k)=\left\{1+K_{15} \frac{v-2}{v-1}(k-1)\right\}^{(v-1) /(v-2)}
$$

so that, by (4.8), $g(e, \lambda(k)) \geq k, 0<e<\infty$. Thus, setting $M^{-1}(s)=e$ and using (4.6) yields

$$
\begin{aligned}
\frac{M^{-1}(k s)}{M^{-1}(s)} & =\frac{M^{-1}(k M(e))}{e} \leq \frac{M^{-1}(g(e, \lambda(k)) M(e))}{e} \\
& =\frac{M^{-1}(M(\lambda(k) e))}{e}=\lambda(k)
\end{aligned}
$$

which proves $(4.5)$.

\section{REFERENCES}

[1] C. M. Dafermos and L. Hsiao, Global smooth thermomechanical processes in one-dimensional nonlinear thermoviscoelasticity, J. Nonlinear Analysis 6 (1982), 435-454

[2] C. M. Dafermos, Global smooth solutions to the initial-boundary value problem for the equations of onedimensional nonlinear thermoviscoelasticity, SIAM J. Math. Analysis 13 (1982), 397-408 